\title{
Optimization of Atrioventricular Delay and Follow-up in a Patient with Congestive Heart Failure and with Bi-ventricular Pacing
}

\author{
Toshiyuki IshIKAwA, ${ }^{1} \mathrm{MD}$, Shinichi SumitA, ${ }^{1} \mathrm{MD}$, Kazuo KIMURA, ${ }^{1} \mathrm{MD}$, \\ Miyako KIKUCHI, ${ }^{1} \mathrm{MD}$, Kohei Matsushita, ${ }^{1} \mathrm{MD}$, Yasuo OhKusu, ${ }^{1} \mathrm{MD}$, \\ Takeshi NAKAgAwA, ${ }^{1}$ MD, Masami Kosuge, ${ }^{1}$ MD, Takashi Usui, ${ }^{1}$ MD, \\ and Satoshi UMEMURA, ${ }^{1} \mathrm{MD}$
}

\begin{abstract}
SUMMARY
Cardiac function is improved by bi-ventricular pacing in patients with severe reduced cardiac function. Atrioventricular (AV) delay optimization is also important in this therapy. However, the AV delay required to achieve the optimal AV synchrony varied from time to time. We have reported that the critical AV delay that induces diastolic mitral regurgitation (MR) may represent the upper limit of the optimal AV delay. The optimal $\mathrm{AV}$ delay can be predicted by a simple method; slightly prolonged AV delay-interval between the end of atrial kick and complete closure of the mitral valve (duration of diastolic MR) at the AV delay setting. [Case] 60 year old Japanese male with dilated cardiomyopathy. He was repeatedly admitted to our hospital due to congestive heart failure. Ejection fraction was $14 \%$. ECG showed complete left bundle branch block and his PQ interval was $0.22 \mathrm{sec}$. He was dependent on intravenous injections of catecolamine and could not be discharged from the hospital for over one year. Optimal AV delay was predicted as $80 \mathrm{msec}$ during bi-ventricular pacing by our formula. Cardiac output was 4.9 , 6.0, 5.1 l//min when the AV delay was set at 50, 80, $110 \mathrm{msec}$. Cardiac function was improved from NYHA class III to IIand he has been relieved from the dependency on intravenous catecholamine injections. AV delay was optimized $(70-100 \mathrm{msec})$ by our method during follow-up for one year. This case indicates that AV delay optimization is important in bi-ventricular pacing. (Jpn Heart J 2001; 42: 781-787)
\end{abstract}

Key words: Dilated cardiomyopathy, DDD pacemaker, Multisite pacing, Atrioventricular delay, Doppler echocardiography

THE efficacy of a short atrio-ventricular (AV) delay in patients with severely impaired cardiac function has been reported. ${ }^{1-4)}$ However, right ventricular pacing during $\mathrm{AV}$ sequential pacing has deleterious effects. ${ }^{5}$ It has been reported that biventricular pacing can improve hemodynamics in patients with congestive heart

From ${ }^{1}$ the Second Department of Internal Medicine, Yokohama City University School of Medicine, Yokohama, Japan.

Address for correspondence: Toshiyuki Ishikawa, MD, Second Department of Internal Medicine, Yokohama City University School of Medicine, 3-9, Fukuura, Kanazawa-ku, Yokohama 236-0004, Japan.

Received for publication April 16, 2001.

Revised and accepted June 18, 2001. 
failure. ${ }^{6,7)}$ Both AV delay optimization and shortening of the ventricular activation sequence may be important in patients with severely impaired cardiac function, and AV delay optimization may also be important during bi-ventricular pacing. However, the optimal AV delay may not be a fixed value in each patient, and thus should be adjusted constantly.

Diastolic mitral regurgitation (MR) is observed in patients with elevated left ventricular end-diastolic pressure ${ }^{8)}$ and can be induced by prolonging the AV delay in patients with DDD pacemakers. ${ }^{5,8-15)}$ Cardiac function may be improved by AV sequential pacing and setting the AV delay under the critical PQ interval for the appearance of diastolic MR when the diastolic MR is observed in patients with intrinsic AV conduction. ${ }^{4,5}$ We have determined a new formula for predicting the optimal AV delay using Doppler echocardiography; optimal AV delay=slightly prolonged AV delay-interval between end of atrial kick and complete closure of the mitral valve (duration of diastolic MR) at the AV delay setting (Figure 1). ${ }^{12)}$

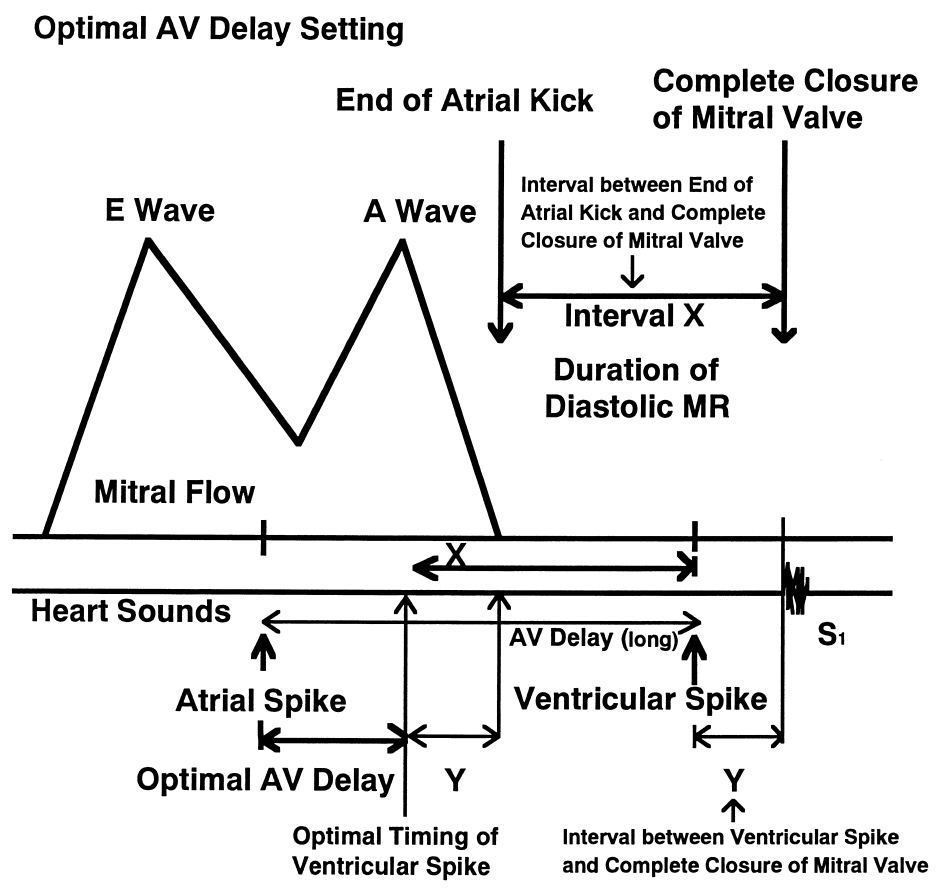

Figure 1. Optimal atrioventricular (AV) delay setting. Atrial kick may be interrupted by a too short AV delay, and filling time may be shortened by a too long AV delay in patients with an implanted DDD pacemaker. The optimal AV delay allows completion of end-diastolic filling flow prior to ventricular contraction, providing the longest diastolic filling time. The AV delay at which the end of the A wave on transmitral flow coincides with complete closure of the mitral valve is optimal. AV delay (long) is optimal AV delay + interval between ventricular spike and complete closure of mitral valve caused by ventricular contraction (Interval Y) + interval between end of atrial kick and ventricular spike. The optimal AV delay can be predicted by a simple method; slightly prolonged AV delay-interval between the end of atrial kick and complete closure of the mitral valve (Interval X; duration of diastolic MR) at the AV delay setting. 
We report a patient with severe congestive heart failure who remained in good condition over one year by bi-ventricular pacemaker implantation and $\mathrm{AV}$ delay optimization.

\section{CASE RePORT}

The patient was a 60 year old Japanese male with dilated cardiomyopathy. He had bronchial asthma and $10 \mathrm{mg}$ per day oral administration of predonisolone was required. He had ventricular tachycardia and was treated with oral amiodarone at a dose of $100 \mathrm{mg}$ a day. He was repeatedly admitted to our hospital due to congestive heart failure 6 times over 5 years. He was admitted to our hospital due to heart failure on January 1, 1999. Ejection fraction was $14 \%$ by echocardio-
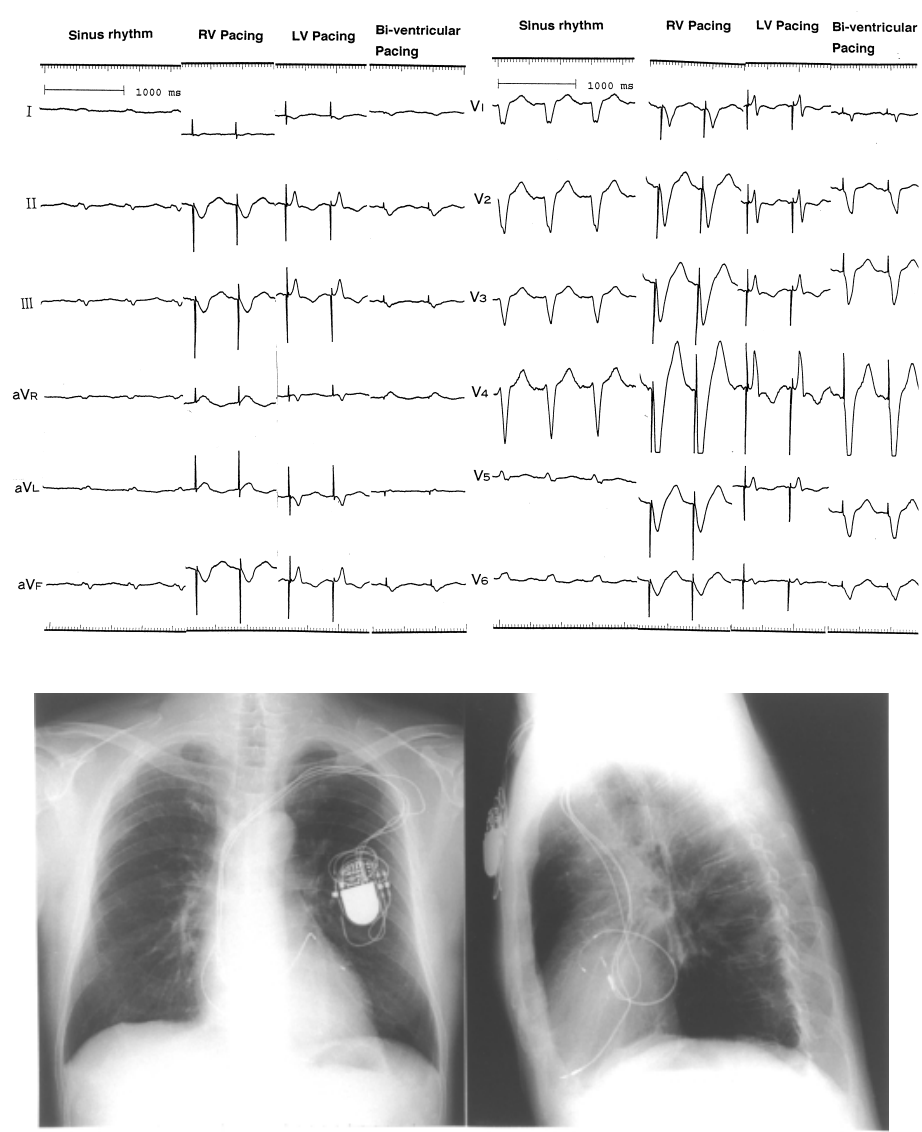

Figure 2. ECG showed prolongation of PQ interval $(0.21 \mathrm{sec})$ and complete left bundle branch block with wide QRS complex (172 msec) (A). The left ventricular pacing lead was positioned at the great cardiac vein through the coronary sinus (B). The QRS complex durations were $192 \mathrm{msec}$ during right ventricular pacing, $172 \mathrm{msec}$ during left ventricular pacing, and $165 \mathrm{msec}$ during bi-ventricular pacing (A). After bi-ventricular pacing, cardiac function was improved from New York Heart Association class III to II without intravenous injection of catecholamine (B). 
graphy and the cardiothoracic ratio (CTR) on chest XP was 61\%. ECG showed complete left bundle branch block. QRS duration was $172 \mathrm{msec}$ and PQ interval was $0.22 \mathrm{sec}$ (Figure 2A). He was treated with oral furosemide $(120 \mathrm{mg})$, spironolactone $(25 \mathrm{mg})$, digoxine $(0.1 \mathrm{mg})$, amiodarone $(100 \mathrm{mg})$, losartan potassium $(12.5 \mathrm{mg})$, isosorbide mononitrate $(60 \mathrm{mg})$, docarpamine $(2.25 \mathrm{~g})$, and denopamine $(30 \mathrm{mg}) . \beta$-Blockers could not be used due to bronchial asthma. Additionally, he was dependent on intravenous injections of catecolamine (dopamine hydrochloride, $0.003 \mathrm{mg} / \mathrm{kg} / \mathrm{min}$ ) and could not be discharged from the hospital for over one year.

A DDD pacemaker with a bi-ventricular pacing system was implanted on December 17, 1999. As there were only small lateral branches of the coronary sinus by retrograde coronary sinus venography, a left ventricular pacing lead was positioned at the great cardiac vein through the coronary sinus. Another unipolar lead was positioned at the right ventricular apex. A J-shaped lead was positioned at the right atrial appendage for right atrial pacing. The QRS complex durations were $192 \mathrm{msec}$ during right ventricular pacing, $172 \mathrm{msec}$ during left ventricular pacing, and $165 \mathrm{msec}$ during bi-ventricular pacing (Figure 2A). The left ventricular lead (distal portion; -) and right ventricular lead (proximal portion; +) were connected by a Y-connector (Figure 2B). The pacing threshold for bi-ventricular pacing was $3.5 \mathrm{~V}(1.0 \mathrm{msec})$, the sensing threshold for bi-ventricular pacing was $\geqq 20 \mathrm{mV}$, and lead impedance was $3000 \Omega$

Optimal AV delay was predicted as $80 \mathrm{msec}$ during bi-ventricular pacing by our formula (Figure 3). Cardiac output was $4.2 \mathrm{l} / \mathrm{min}$ and diastolic filling time was $180 \mathrm{msec}$ during 80/min atrial pacing. Cardiac output was 4.9, 6.0, and 5.1 l/ min and diastolic filling time was 305,320 , and $260 \mathrm{msec}$ when the AV delay was set at 50, 80, and $110 \mathrm{msec}$. The optimal AV delay was $30 \mathrm{msec}$ during right ventricular pacing, $50 \mathrm{msec}$ during left ventricular pacing, and $80 \mathrm{msec}$ during biventricular pacing. Cardiac output was 5.2, 5.7, and $6.0 \mathrm{l} / \mathrm{min}$ and diastolic filling time was 285,415 , and $320 \mathrm{msec}$, respectively.

He has been relieved from the dependency on intravenous catecholamine injections. His cardiac function improved from NYHA class III to II (Figure 2b), and he was discharged from the hospital. Oral medications were not changed and AV delay was optimized (70-100 msec) by our method during follow-up for one year. 


\section{Sinus rhythm}

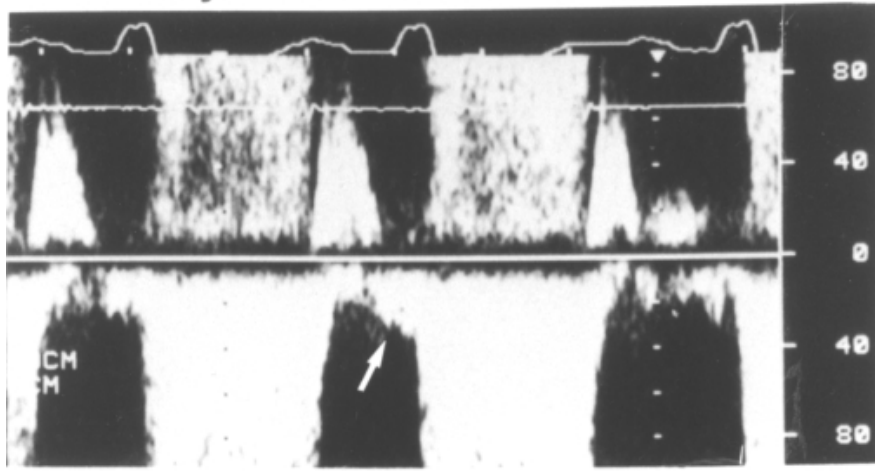

\section{AV delay: $180 \mathrm{msec}$}

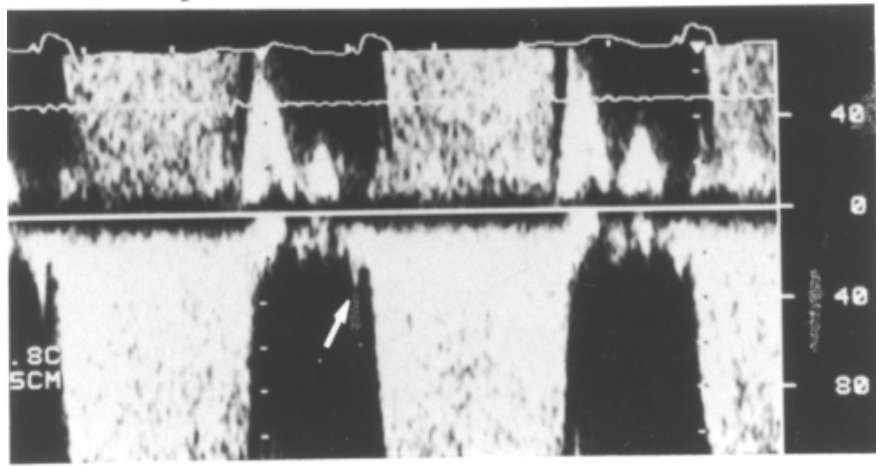

\section{AV delay: $80 \mathrm{msec}$}

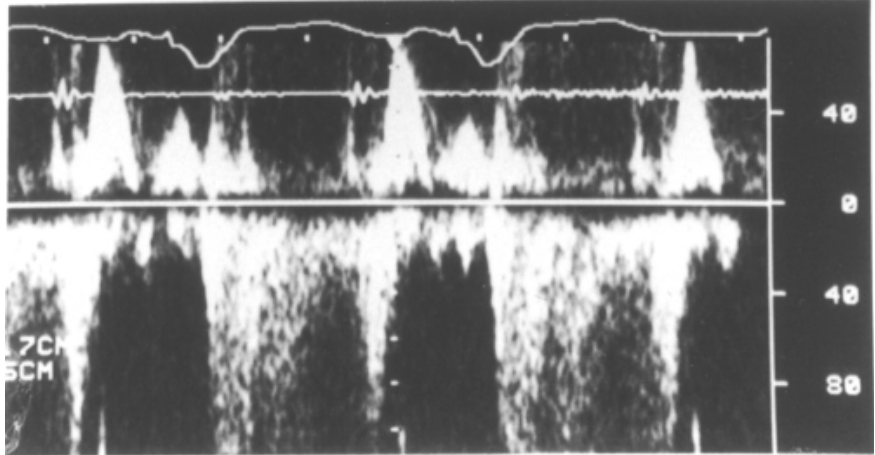

Figure 3. Marked diastolic mitral regurgitation (arrow) was detected (upper panel). During 80 beats/min $\mathrm{AV}$ sequential pacing, diastolic mitral regurgitation was observed when the AV delay was set at $180 \mathrm{msec}$ (arrow). The interval between the end of atrial kick and complete closure of the mitral valve (duration of diastolic mitral regurgitation) was 100 msec. The predicted optimal AV delay was $180-100=80 \mathrm{msec}$ (middle panel). Diastolic mitral regurgitation disappeared when the AV delay was set at $80 \mathrm{msec}$ (lower panel). 


\section{Discussion}

Recently, the efficacy of a short AV delay in patients with severely impaired cardiac function has been reported. ${ }^{1-4)}$ DDD pacing and AV delay optimization is not always effective for congestive heart failure. Right ventricular pacing during AV sequential pacing has deleterious effects. ${ }^{5}$ It has been reported that bi-ventricular pacing can improve hemodynamics in patients with congestive heart failure. $^{6,7)}$ The importance of optimizing AV delay is still controversial ${ }^{1-4,16)}$ in DDD pacing for congestive heart failure. The optimal AV delay may change from time to time with changes in cardiac function. However, AV delay was fixed in previous reports. ${ }^{1,216)}$ The optimal AV delay may not be a fixed value in each patient, and thus should be adjusted constantly. Both AV delay optimization and shortening of the ventricular activation sequence may be important in patients with severely impaired cardiac function, and AV delay optimization may also be important during bi-ventricular pacing.

In patients with an implanted DDD pacemaker, atrial kick may be interrupted by a too short AV delay, and filling time may be shortened by a too long AV delay. The optimal AV delay allows completion of end-diastolic filling flow prior to ventricular contraction, providing the longest diastolic filling time. ${ }^{17)}$ The AV delay at which the end of the A wave on transmitral flow coincides with complete closure of the mitral valve may be optimal. ${ }^{15}$ )

In our previous studies, diastolic mitral regurgitation was detected during the interval between the end of the A wave and complete closure of the mitral valve, and the critical PQ interval that induces diastolic mitral regurgitation may represent the upper limit of the optimal PQ interval. ${ }^{5,8-15)}$ The optimal AV delay can be predicted by a simple method; slightly prolonged AV delay-interval between the end of atrial kick and complete closure of the mitral valve (duration of diastolic MR) at the AV delay setting. ${ }^{15)}$

Bi-ventricular pacing is not always effective for congestive heart failure. The indication for this therapy remains unclear. This patient had a prolonged PQ interval and complete left bundle branch block with a markedly prolonged QRS complex. Therefore, this case was a good candidate for bi-ventricular pacing. Moreover, the AV delay setting could be easily optimized by our new method during follow-up. He remained in NYHA II for over one year. DDD pacing with AV delay optimization alone was effective for this patient. However, the duration of the QRS complex was prolonged from 172 to $192 \mathrm{msec}$ by right ventricular pacing. The duration of the QRS complex was shortened to $165 \mathrm{msec}$ by bi-ventricular pacing and further improvement of the cardiac function has been achieved by bi-ventricular pacing. This experience indicated that bi-ventricular pacing and AV delay optimization was useful for patients with severe congestive 
heart failure.

Although hemodynamic assessment by catheter examination provides the most reliable measurements of cardiac output and intracardiac pressures, it is an invasive procedure. Our new method is easy and not time-consuming. Thus, AV delay optimization can be easily repeated. This method is useful for the follow-up of patients with congestive heart failure and with bi-ventricular pacing. In conclusion, AV delay optimization during bi-ventricular pacing is important in patients with severe congestive heart failure.

\section{REFERENCES}

1. Hochleitner M, Hörtangl H, $\mathrm{NgCK}$, et al. Usefulness of physiologic dual chamber pacing in drug-resistant idiopathic dilated cardiomyopathy. Am J Cardiol 1990; 66: 198-202.

2. Auricchio A, Sommariva L, Salo RW, et al. Improvement of cardiac function in patients with severe congestive heart failure and coronary artery disease by dual chamber pacing with shortened AV delay. PACE 1993; 16: 2034-43.

3. Nishimura R, Hayes DL, Holmes DR, et al. Mechanism of hemodynamic improvement by dual-chamber pacing for severe left ventricular dysfunction: an acute Doppler and catheterization hemodynamic study. JACC 1995; $25: 281-8$.

4. Ishikawa T, Sumita S, Kosuge M, et al. Optimization of atrioventricular delay and follow-up in a patient with congestive heart failure with an implanted DDD pacemaker -Case report- Jpn Circ J 2001; 65: 46-9.

5. Ishikawa T, Sumita S, Kimura K, et al. Efficacy of atrioventricular sequential pacing and diastolic mitral regurgitation in patients with intrinsic atrioventricular conduction. Jpn Circ J 2000; 64: 579-82.

6. Cazeau S, Ritter P, Lazarus A, et al. Multisite pacing for end-stage heart failure: early experience. PACE 1996; 19: 1748-57.

7. Grass D, Mabo F, Tang T, et al. Multi-site pacing as a congestive heart failure: Preliminary results of the Medtronic Inc. InSync study. PACE 1998; 21: 2249-55.

8. Ishikawa T, Usui T, Kashiwagi M, et al. Contribution of end-diastolic mitral regurgitation to the B-B' step formation on M-mode echocardiography. J Appl Cardiol 1991; 6: 163-8.

9. Ishikawa T, Kimura K, Nihei T, et al. Relationship between diastolic mitral regurgitation and PQ interval or cardiac function in patients implanted with DDD pacemakers. PACE 1991; 14: 1797-902.

10. Ishikawa T, Kimura K, Miyazaki N, et al. Diastolic mitral regurgitation in patients with first-degree atrioventricular block. PACE 1992; 15: 1927-31.

11. Ishikawa T, Sumita S, Kimura K, et al. PQ interval and diastolic mitral regurgitation in patients with sick sinus syndrome. Eur J Cardiac Pacing Electrophysiol 1994; 4: 170-5.

12. Ishikawa T, Sumita S, Kimura K, et al. Critical PQ interval for the appearance of diastolic mitral regurgitation and optimal PQ interval in patients implanted with DDD pacemakers. PACE 1994; 17: 1989-94.

13. Ishikawa T, Sumita, Kikuchi M, et al. Diastolic mitral regurgitation when the heart rate is normalized by ventricular pacing. Eur J Cardiac Pacing Electrophysiol 1996; 6: 23-7.

14. Ishikawa T, Sumita S, Kimura K, et al. Optimal atrioventricular setting and diastolic mitral regurgitation in patients implanted with DDD pacemakers. Eur J Cardiac Pacing Electrophysiol 1996; 6: 159-63.

15. Ishikawa T, Sumita S, Kimura K, et al. Prediction of optimal atrioventricular delay in patients with implanted DDD pacemakers. PACE 1999; 22: 1365-71.

16. Brecker SJD, Xiao HB, Sparrow J. Effects of dual-chamber pacing with short atrioventricular delay in dilated cardiomyopathy. Lancet 1992; 340: 1308-12.

17. Ritter P, Dib JC, Mahaux V, Leliève T, et al. New method for determining the optimal atrio-ventricular delay in patients paced in DDD mode for complete atrio-ventricular block (abstract). PACE 1995; 18: 855. 